\title{
EUS-directed transgastric endoscopic retrograde cholangiopan- creatography versus laparoscopic-assisted ERCP versus deep enteroscopy-assisted ERCP for patients with RYGB
}

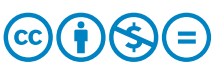

\author{
Authors \\ Manish Dhawan ${ }^{1}$, Shyam Thakkar ${ }^{1}$ \\ Institutions \\ 1 Division of Gastroenterology and Hepatology, Allegheny \\ Health Network, Pittsburgh, Pennsylvania, United States \\ 2 Medicine Institute, Allegheny Health Network, \\ Pittsburgh, Pennsylvania, United States
}

Gursimran S. Kochhar ${ }^{1}$, Nabeeha Mohy-ud-din², Abhinav Grover ${ }^{1}$, Neil Carleton ${ }^{1}$, Abhijit Kulkarni ${ }^{1}, K^{2}$ atie Farah ${ }^{1}$,

submitted 6.1.2020

accepted after revision 30.3 .2020

\author{
Bibliography \\ DOI https://doi.org/10.1055/a-1164-6282 | \\ Endoscopy International Open 2020; 08: E877-E882 \\ (c) Georg Thieme Verlag KG Stuttgart · New York \\ eISSN 2196-9736 \\ Corresponding author \\ Gursimran S. Kochhar, MD, 1307 Federal Street, \\ Suite B-100, Pittsburgh, PA, USA, 15212 \\ Fax: 412-359-8955 \\ gursimran.kochhar@ahn.org
}

\section{ABSTRACT}

Background and study aims Endoscopic ultrasound-directed transgastric endoscopic retrograde cholangiopancreatography (ERCP) (EDGE) is a novel technique for managing pancreaticobiliary diseases in patients with a history of
Roux-en-Y Gastric Bypass (RYGB). It has shown to have high technical success rates and fewer adverse events as compared to laparoscopic-assisted ERCP (LA-ERCP). We compared the technical success and clinical outcomes of EDGE vs. LA-ERCP vs. E-ERCP.

Patients and methods $A$ retrospective chart review was performed for 56 patients, of whom 18 underwent LAERCP, 12 underwent E-ERCP, and 26 had EDGE, and a comparison of technical success and complication rates was done.

Results Baseline demographic characteristics of patients undergoing these procedures, including age and gender, were comparable. The technical success rate for patients in the EDGE group were $100 \%(n=26)$, compared with $94 \%$ $(n=17)$ and $75 \%(n=9)$ in the LA-ERCP and E-ERCP groups $(P=0.02)$. In the EDGE group, $8 \%$ of patients $(n=2)$ had bleeding, and $4 \%$ of patients $(n=1)$ had lumen-apposing metal stent migration occur during the procedure. In the LA-ERCP group $6 \%(n=1)$ of patient had bleeding, $6 \%$ $(n=1)$ post-ERCP pancreatitis and $6 \%(n=1)$ were diagnosed with an intra-abdominal infection post-procedure. Time to complete the EDGE procedure was significantly shorter at $79 \pm 31$ mins, compared with $158 \pm 50$ mins for LA-ERCP and $102 \pm 43$ mins for E-ERCP $(P<0.001)$.

Conclusion EDGE is a novel procedure with short procedure times and an effective alternative to LA-ERCP and EERCP in management of pancreaticobiliary diseases in patients with a history of RYGB.

\section{Introduction}

Endoscopic ultrasound-directed transgastric Endoscopic Retrograde Cholangiopancreatography (EDGE) is a novel technique for managing pancreaticobiliary diseases in patients with a history of Roux-en-Y Gastric Bypass (RYGB). Obesity affects about 93.3 million US adults, and in 2017, there were 228,000 total bariatric procedures $[1,2]$. It is estimated that up to $50 \%$ of patients who have undergone RYGB develop gallstone disease [3]. Patients with altered anatomy can pose a challenge to endos- copists and performing a diagnostic or therapeutic procedure can be difficult.

Laparoscopic-assisted endoscopic retrograde cholangiopancreatography (LA-ERCP) is the current method of choice in most centers. However, it is a time-consuming, invasive procedure that requires coordination between endoscopists and surgeons. Even though reported technical success rates are high, LA-ERCP can have adverse events (AEs) in addition to those incurred with ERCP. In a study by Grimes et al., 84 cases of LA-ERCP were reviewed, out of which bowel injury occurred in $5.9 \%$ cases, access site infection occurred in $4.8 \%$, and $5 \%$ of 
cases had to be converted to open procedures [4]. Enteroscopy-assisted ERCP (E-ERCP) is being performed routinely by endoscopists; however, this has low reported success rates [5]. The success of E-ERCP is dependent on the length of the roux limb, which in some cases, is not available or is not known. Moreover, expertise in performing a single and/or double balloon enteroscopy may also not be available at all endoscopic centers.

In comparison to the above, EDGE is a novel technique that involves placement of a transgastric or trans jejunal lumen-apposing metal stent (LAMS) to create a fistula, through which an ERCP can then be performed. This procedure can be performed in a single step on the same day, or the ERCP can be performed later after a fistula accessing the remnant stomach has been created. Kedia et al. first described this procedure in a video explaining the performance of EDGE in 6 patients with RYGB anatomy with biliary disease [6]. A recent study performed by James $\mathrm{H}$ ] et al. compared the cost-effectiveness of EDGE with deviceassisted ERCP and L-ERCP and concluded that EDGE was the most cost-effective modality [7].

The purpose of our study was to evaluate technical success and clinical outcomes of EDGE vs. LA-ERCP vs. E-ERCP to provide more information to endoscopists who are frequently faced with the decision of offering the most appropriate procedure to their patients.

\section{Patients and methods}

\section{Study design}

A retrospective chart review was performed for patients who underwent LA-ERCP, E-ERCP, or EDGE at a tertiary care hospital in Pennsylvania between January 2015 and July 2019. The study was part of a quality improvement project; hence this project received an IRB no purview determination (exemption) indicating that this is not considered human subject's research. A formal written consent was not required as part of this chart review.

\section{Inclusion criteria}

All adult patients (age $>18$ years) with a history of RYGB who underwent therapeutic ERCP at our tertiary care center were included. As this was a retrospective study, patient allocation was random, based on informed discussions between performing endoscopist and the patients at the time of the procedure regarding risks and benefits of each procedure.

\section{Exclusion criteria}

Patients aged less than 18 years and pregnant patients were excluded.

\section{Data collection}

Patient demographics, including age and gender, clinical parameters such as aspartate aminotransferase, alanine aminotransferase, alkaline phosphatase, total bilirubin, prior imaging, procedural details, and clinical outcomes (technical success and complication rates) were recorded for each treatment group.

\section{Outcomes}

The primary outcomes were technical success with the respective procedure, defined as cannulation of the intended duct. The following were the secondary outcomes for the study:

- Difficulty of ERCP procedure was defined as the level of difficulty documented by performing endoscopist or described as difficult if there was a performance of precut sphincterotomy or $>2$ attempted cannulations of the intended duct. This definition of difficulty is in accordance with previously published manuscripts that have used similar definitions [8].

- Time to complete procedure for EDGE was defined as the sum of time taken for initial EUS to form a fistula and the time taken to perform the ERCP if both procedures were not performed on the same day. Time for LA-ERCP and E-ERCP was calculated as the time from the start to the end of the procedure as documented in the anesthesia notes.

- Length of hospital stay was calculated as the number of days until the patient was discharged from the hospital after the procedure was performed.

- AEs related to the procedure were defined as bleeding either during or up to 7 days after the procedure, post-ERCP pancreatitis, perforation, or infection.

- Infection was determined to be present if the patient became febrile or had positive blood cultures requiring antibiotics after the procedure.

\section{Technique}

The EDGE procedure can be performed as one stage or a twostage procedure, i.e., access to the excluded stomach through a LAMS and an ERCP can be completed in the same endoscopic session, or the ERCP can be performed in a different endoscopic session [6,9]. At our center, the EDGE procedure is performed in a standard fashion by our advanced endoscopists (GK, MD, ST), in either a single or two-stage endoscopic session. Currently, a decision about whether a single or a two-stage procedure is performed is based on three factors. If the patient clinically has acute cholangitis, and they are agreeable to EDGE procedure then consideration is given to a single stage EDGE procedure. The endoscopist's preference, depending on his or her prior experience and comfort level is also considered. Finally, patient preference, that is, if the patient cannot or does not want to return for a second procedure, is considered.

First, using a linear EUS Endoscope (Olympus, GF-UCT180), the excluded stomach is visualized by the identification of gastric rugae ( $\mathbf{F i g}$. 1a). Then a 19-G needle is used to puncture the excluded stomach, and a 0.025 -inch soft jag wire is placed through the needle. Next, mixture of contrast along with saline is injected into the stomach to distend the stomach and visualize the antrum and pylorus ( $\triangleright$ Fig. $\mathbf{1 b}$ ). Once the native stomach is adequately visualized, a LAMS delivery system is advanced over the guidewire. At our center, we prefer using a guidewire versus a free hand technique for placement of LAMS as we believe this is a relatively safer approach to access the native stomach.

Using a Hot AXIOS delivery system (Boston Scientific, Marlborough, Massachusetts, United States), a fistula is made be- 

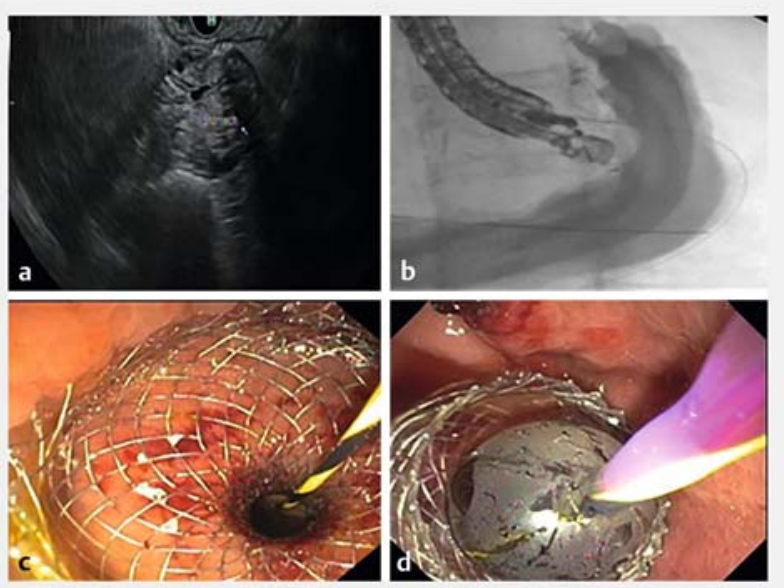

- Fig. 1 a Visualization of excluded stomach through endoscopic ultrasound. b Fluoroscopic imaging showing distension of stomach after injection of contrast. c Deployment of LAMS after creation of a gastro-gastric/gastro-jejunal fistula. $\mathbf{d}$ Dilation of LAMS with a CRE Balloon.

tween the gastric pouch (or remnant jejunum) and the excluded stomach, and the LAMS is deployed under EUS guidance ( Fig. 1c). The stent tract is dilated with a dilating balloon, to either 15 or $20 \mathrm{~mm}$, depending on the size of the LAMS used ( $\triangleright$ Fig.1d). The EUS scope and the guidewire are then withdrawn. A duodenal scope (Olympus endoscopy) is then advanced through the oropharynx, across the fistula, through the LAMS into the excluded stomach. It is then advanced to the area of the papilla, and a standard ERCP is performed. The LAMS is left in place for 7-10 days and if no further need of
ERCP then, LAMS is removed via esophagogastroduodenoscopy (EGD) using rat-toothed forceps.

\section{Statistical analysis}

The continuous variables were reported as means with standard deviations, whereas proportions were reported as percentages. Statistical analysis was performed using statistical package for social sciences (SPSS) 26.0 software (SPSS Inc., Chicago Illinois, United States). For comparison of continuous variables among treatment arms, unpaired student t-test, and analysis of variance (ANOVA) test was used. For comparison of categorical variables, chi-square and Fisher exact tests were used. $P<0.05$ was considered significant.

\section{Results}

A total of 56 patients met our inclusion criteria and were included in the study. Of them, 26 patients underwent EDGE, 18 underwent LA-ERCP, and 12 underwent E-ERCP. Baseline characteristics of patients, including age and gender, were not statistically different. ERCP was performed after a mean of 10.7 years following bariatric surgery for EDGE, LA-ERCP patients, and EERCP patients $(P=0.188)$. Baseline characteristics, including liver function tests and imaging performed before ERCP, are detailed in $>$ Table 1 .

Indications for the procedure were also comparable across all groups. Thirty-eight percent of patients $(n=10)$ in the EDGE group had ERCP performed for choledocholithiasis, compared with $78 \%(n=14)$ and $42 \%(n=5)$ for LA-ERCP and E-ERCP, respectively $(P=0.37)$. Indications for the procedures are detailed in $>$ Table 2. The technical success rate for patients in the EDGE group was $100 \%(n=26)$, compared with $94 \%(n=$ $17)$ and $75 \%(\mathrm{n}=9)$ in the LA-ERCP and E-ERCP groups ( $P=$

- Table 1 Demographics and baseline liver function tests and imaging performed prior to EDGE, LA-ERCP and E-ERCP.

\begin{tabular}{|c|c|c|c|c|}
\hline & EDGE $(n=26)$ & LA-ERCP $(n=18)$ & E-ERCP $(n=12)$ & $P$ value \\
\hline Age (mean \pm S.D.) & $60.77 \pm 11.44$ & $60.78 \pm 12.67$ & $68.58 \pm 15.09$ & 0.18 \\
\hline \multicolumn{5}{|l|}{ Gender } \\
\hline Female (n, \%) & $20(77)$ & $12(67)$ & $8(67)$ & \multirow[t]{2}{*}{0.70} \\
\hline Male (n, \%) & $6(23)$ & $6(33)$ & $4(33)$ & \\
\hline AST & $49 \pm 35$ & $172 \pm 268$ & $77 \pm 66$ & 0.04 \\
\hline ALT & $65 \pm 63$ & $197 \pm 276$ & $114 \pm 113$ & 0.05 \\
\hline Alkaline Phosphatase & $231 \pm 176$ & $355 \pm 415$ & $290 \pm 275$ & 0.40 \\
\hline Total Bilirubin & $2.0 \pm 4.0$ & $2.5 \pm 2.8$ & $2.29 \pm 2.43$ & 0.88 \\
\hline \multicolumn{5}{|l|}{ Prior Imaging (n, \%) } \\
\hline CT Abdomen Pelvis & $46(12)$ & $7(39)$ & $4(33)$ & \multirow[t]{3}{*}{0.53} \\
\hline MRCP & $12(46)$ & $9(50)$ & $6(50)$ & \\
\hline Previous ERCP & $1(4)$ & $0(0)$ & $0(0)$ & \\
\hline
\end{tabular}


- Table 2 Indication for EDGE, LA-ERCP and E-ERCP.

\begin{tabular}{|c|c|c|c|c|}
\hline Indication ${ }^{1}$ & EDGE $(n=26)$ & LA-ERCP $(n=18)$ & E-ERCP $(n=12)$ & $P$ value \\
\hline Choledocholithiasis (n, \%) & $10(38)$ & $14(78)$ & $5(42)$ & \multirow[t]{9}{*}{0.37} \\
\hline Abdominal pain, LFT abnormalities and CBD Dilation on imaging (n, \%) & $5(19)$ & $0(0)$ & $1(8)$ & \\
\hline LFT abnormalities with CBD dilation on imaging ( $\mathrm{n}, \%$ ) & $0(0)$ & $1(5.5)$ & $0(0)$ & \\
\hline Pancreatic duct dilation ( $\mathrm{n}, \%$ ) & $2(8)$ & $0(0)$ & $0(0)$ & \\
\hline Biliary sludge or biliary leak (n, \%) & $3(8.3)$ & $1(5)$ & $1(8)$ & \\
\hline Biliary stricture (n, \%) & $3(8.3)$ & $0(0)$ & $2(17)$ & \\
\hline Cholangitis (n, \%) & $1(4)$ & $1(5)$ & $2(17)$ & \\
\hline Pancreatitis (n, \%) & $2(8)$ & $2(11)$ & $2(17)$ & \\
\hline Others (n, \%) & $0(0)$ & $2(11)$ & $0(0)$ & \\
\hline \multicolumn{5}{|c|}{$\begin{array}{l}\text { Others: intrahepatic biliary ductal dilation on imaging and papillary stenosis } \\
\text { EDGE, endoscopic ultrasound-directed transgastric endoscopic retrograde cholangiopancreatography; LA-ERCP, laparoscopy-assisted etrograde cholangiopancrea- } \\
\text { tography; E-ERCP, enteroscopy-assisted endoscopic retrograde cholangiopancreatography; LFT, liver function test; CBD, common bile duct } \\
1 \text { Procedure may have been performed for multiple indications. }\end{array}$} \\
\hline
\end{tabular}

- Table3 Method of removal of LAMS.

\begin{tabular}{|l|c|}
\hline Method of LAMS removal & EDGE (n=26) \\
\hline During index ERCP $(\mathrm{n}, \%)$ & $8(31)$ \\
\hline Follow-up EGD $(\mathrm{n}, \%)$ & $9(35)$ \\
\hline Intra-operatively $(\mathrm{n}, \%)$ & $3(12)$ \\
\hline Follow-up ERCP & $2(8)$ \\
\hline Follow-up EUS (n, \%) & $1(4)$ \\
\hline Not removed at the time of this study (n, \%) & $3(12)$ \\
\hline $\begin{array}{l}\text { LAMS, lumen-apposing metal stent; EDGE, endoscopic ultrasound-directed } \\
\text { transgastric endoscopic retrograde cholangiopancreatography; ERCP, } \\
\text { endoscopic retrograde cholangiopancreatography; EGD, esophagogastro- } \\
\text { duodenoscopy; EUS, endoscopic ultrasound }\end{array}$ \\
\hline
\end{tabular}

0.02 ). Difficulty of the procedure as defined in Methods was low in $100 \%$ of patients $(n=26)$ in the EDGE group and $78 \%$ of patients $(n=14)$ in the LA-ERCP group. Difficulty of procedure was defined as high in $58 \%$ of patients $(n=7)$ in the E-ERCP group $(P<0.001)$.

In the EDGE group, $92 \%(n=24)$ had a 15 -mm LAMS placed and $8 \%(n=2)$ had a 20 -mm LAMS placed. This was at the discretion of the performing endoscopist. Eight percent of patients $(n=2)$ had sphincterotomy-related bleeding, and $4 \%$ of patient $(n=1)$ had LAMS migration occur during the procedure. In the LA-ERCP group $6 \%$ of patients $(n=1)$ had sphincterotomy-related bleeding, $6 \%(n=1)$ post-ERCP pancreatitis and $6 \%(n=1)$ were diagnosed with an intra-abdominal infection post-procedure. In the E-ERCP group $25 \%$ of patients $(n=3)$ had sphincterotomy-related bleeding, $8 \%(n=1)$ had infection and $8 \%(n=1)$ were diagnosed with post-ERCP pancreatitis.

Time to complete the EDGE procedure was statistically significantly shorter at $79 \pm 31$ mins, compared with $158 \pm 50$ mins for LA-ERCP and $102 \pm 43$ mins for E-ERCP $(P<0.001)$. Hospital length of stay for EDGE was $1.61 \pm 1.74$ days, compared with $2.44 \pm 1.82$ days for LA-ERCP and $3.26 \pm 4.36$ days for E$\operatorname{ERCP}(P=0.18)$.

The EDGE procedure was performed after a failed ERCP for $15 \%$ of patients $(n=4)$. Three of these patients had undergone failed L-ERCP, and one had undergone failed E-ERCP. The access route for EDGE was transgastric in $84.6 \%$ of patients $(n=22)$. EUS with placement of AXIOS stent and ERCP was performed on the same day for $50 \%$ of patients $(n=13)$. LAMS was removed during the index ERCP for $31 \%$ of patients $(n=8)$ and at a follow-up EGD for $35 \%$ of patients $(n=9)$. Details regarding the method of LAMS removal are described in > Table 3.

The gastro-gastric or gastro-jejunal fistula closed spontaneously in $69 \%$ of EDGE cases $(n=18) .12 \%$ of patients $(n=3)$ underwent closure of fistula intra-operatively as part of the performance of a Whipple's procedure. Four percent of patients $(n=1)$ had closure achieved by endoscopic mucosal clips and LAMS was in-situ in $12 \%$ of patients $(n=3)$ at the time of this study. Four percent of patients $(n=1)$ were lost to follow-up. Follow-up methods to ensure closure of fistula are detailed in - Table 4. The average weight change for patients at 11 weeks from the EDGE procedure was $-1.4 \mathrm{kgs}( \pm 6.5)$.

\section{Discussion}

As the proportion of obesity within the population continues to increase, so does the proportion of population undergoing weight-loss surgeries such as RYGB [10]. The presence of pancreaticobiliary diseases in patients who have undergone RYGB has been well-described in the literature [11]. The current standard for treatment for these diseases is LA-ERCP or EERCP; however, with the evolution of interventional endoscopies, E-ERCP has also become a standard procedure. LA-ERCP needs to be performed in the operating room, with creation of a gastrostomy site. It is a time-consuming procedure and presents with risks of bleeding, infection, and perforation; however, it has a near $100 \%$ technical success rate. E-ERCP has a 
Table4 Follow-up methods to determine clinically significant fistula.

\begin{tabular}{|c|c|}
\hline $\begin{array}{l}\text { Follow-up method to determine clinically } \\
\text { significant fistula }\end{array}$ & $\begin{array}{l}\text { EDGE } \\
(n=26)\end{array}$ \\
\hline Clinic visits with weight checks (n, \%) & $12(46)$ \\
\hline Follow-up gastrointestinal series ( $\mathrm{n}, \%$ ) & $5(19)$ \\
\hline $\operatorname{EGD}(n, \%)$ & $3(11.5)$ \\
\hline LAMS in place at study end point ( $\mathrm{n}, \%)$ & $3(11.5)$ \\
\hline Lost to follow-up (n, \%) & $1(4)$ \\
\hline Follow-up scheduled for future at study end point (n,\%) & $2(8)$ \\
\hline \multicolumn{2}{|c|}{$\begin{array}{l}\text { EDGE, endoscopic ultrasound-directed transgastric endoscopic retrograde } \\
\text { cholangiopancreatography; EGD, esophagogastroduodenoscopy; LAMS, } \\
\text { lumen-apposing metal stent }\end{array}$} \\
\hline
\end{tabular}

lower technical success rate than LA-ERCP, less risk of AEs, and is less time-consuming compared to E-ERCP [12]. EDGE carries the benefit of a $100 \%$ technical success rate and can be performed in a shorter time with minimal AEs as described in our study.

An algorithm explaining our current approach in assigning patients to each group is depicted in $\mathbf{F i g}$. 2. A recent meta-analysis comparing EDGE with E-ERCP and LA-ERCP concluded that the technical and clinical success of EDGE is superior to EERCP and comparable to LA-ERCP [13]. Our study provides further comprehensive comparison of outcomes data at a single academic center, thereby allowing gastroenterologists to make educated decisions along with their patients regarding the choice of procedure. Kedia et al. compared 29 cases of EDGE with 43 cases of LA-ERCP and concluded that EDGE is non-inferior to LA-ERCP and safer in comparison [14]. In their study, two cases of bowel perforation occurred in the LA-ERCP group, whereas one happened in the EDGE group. In the EDGE cases, 3 to 4 weeks were allowed between creation of the fistula and performance of ERCP for the maturation of the gastrogastric of jejuno-gastric fistula. Their study was in line with our results, where a concern for weight gain was addressed; however, the final results showed that the mean change in weight was negative $6.6 \mathrm{lb}$ at 28 weeks.

Another study performed by Kedia et al. looked at performance of EDGE procedures in six cases; however, all six cases were performed as two-step procedures [15]. A recent study conducted by James et al. looked at 19 EDGE procedures, in which fistula closure was promoted using argon plasma coagulation (APC) in 12 patients [16]. Eleven patients had upper gastrointestinal series to assess fistula closure, and one persistent fistula was closed endoscopically.

Our study highlights the significantly shorter time to completion of the procedure for EDGE as compared to LA-ERCP and E-ERCP. It has also described a $100 \%$ technical success rate for EDGE. It is noted that in our study, $38 \%$ of patients $(n=10)$ in the EDGE arm underwent the procedure due to an indication of choledocholithiasis, however, this was without statistical significance as compared to the LA-ERCP and E-ERCP arms. Postsphincterotomy bleeding occurred in two patients during the EDGE procedure, and was successfully managed by balloon tamponade with no further bleeding noted. One patient had dislodgement of LAMS after ERCP. The LAMS $(15 \mathrm{~mm} \times 10 \mathrm{~mm}$ AXIOS) was removed in this case, and the fistula was successfully stented again with another LAMS. In the initial stage, 19\% of patients $(n=5)$ in the EDGE group underwent an upper gastrointestinal series to document closure of the fistula when we started performing the procedure at our center. However, when the upper gastrointestinal series in all five cases showed no persistence of fistulas, we then adopted a more cost-effective approach by following patients in clinic with regular weight checks. $54 \%$ of patients $(n=14)$ were followed clinically with regular weight checks to ensure no weight gain.

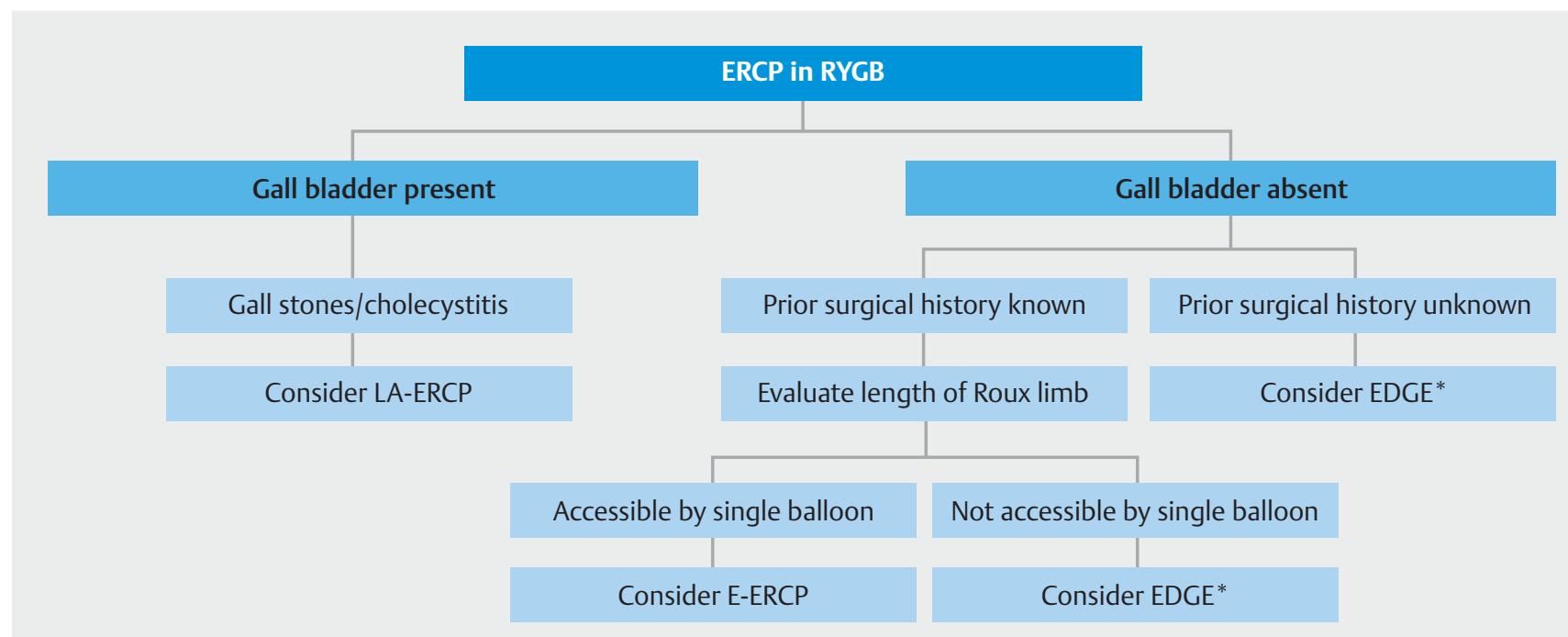

- Fig. 2 Algorithm showing suggested approach to performing ERCP in patients with RYGB. *After having an informed discussion with the patient regarding all 3 modalities of treatment. 
The average weight change at 81 days post-procedure was negative $3.02 \mathrm{lb}$. In addition, three patients in our study had LAMS in place at the study endpoint. Indications for this were need for recurrent ERCPs due to a biliary stricture of unknown etiology in one patient, need for recurrent ERCPs due to locally advanced pancreatic cancer leading to recurrent cholangitis in 1onepatient, and pancreas divisum leading to recurrent pancreatitis in one patient.

Despite the high technical success rate, our study has a few limitations. To begin with, our study was retrospective and from a single center carrying with it all the potential shortcomings of any retrospective study. Although our study had 26 patients who underwent EDGE at a single center, we still had a relatively small number of subjects in the LA-ERCP and E-ERCP groups, thereby limiting some of the data analysis. Also, we could not conclusively document closure of fistulas, and used weight gain as a surrogate marker to ascertain this. We did not note any persistent fistulas in our study; however, longer follow-up and more patients are needed to study that further. Another limitation of this study was that we were unable to define the difficulty level of the EUS portion of EDGE due to the study's retrospective nature. We obtained difficulty level only for the ERCP portion, as described above in Methods.

\section{Conclusion}

The EDGE procedure is a novel technique for management of pancreaticobiliary diseases in patients with RYGB. Based on our initial experience and other studies published in the literature, EDGE should be considered as an alternative to LA-ERCP and E-ERCP to manage hepatopancreaticobiliary complications in patients with RYGB in centers with expertise available.

\section{Competing interests}

The authors declare that they have no conflict of interest.

\section{References}

[1] Adult obesity facts: Overweight \& obesity. Centers for Disease Control and Prevention; https://www.cdc.gov/obesity/data/adult.html

[2] Estimate of bariatric surgery numbers, 2011-2017. American Society for Metabolic and Bariatric Surgery; https://asmbs.org/resources/estimate-of-bariatric-surgery-numbers
[3] Hamdan K, Somers S, Chand M. Management of late postoperative complications of bariatric surgery. $\mathrm{Br}$ J Surg 2011; 98: 1345-1355

[4] Complications of laparoscopic transgastric ERCP in patients with Roux-en-Y gastric bypass. SAGES abstract archives; https://www.sages.org/meetings/annual-meeting/abstracts-archive/complicationsof-laparoscopic-transgastric-ercp-in-patients-with-roux-en-y-gastric-bypass/

[5] Shah RJ, Smolkin M, Yen R et al. A multicenter, U.S. experience of single-balloon, double-balloon, and rotational overtube-assisted enteroscopy ERCP in patients with surgically altered pancreaticobiliary anatomy (with video). Gastrointest Endosc 2013; 77: 593-600

[6] Kedia P, Kumta NA, Sharaiha R et al. Bypassing the bypass: EUS-directed transgastric ERCP for Roux-en-Y anatomy. Gastrointest Endosc 2015; 81: 223-224

[7] James H], James TW, Wheeler SB et al. Cost-effectiveness of endoscopic ultrasound-directed transgastric ERCP compared with deviceassisted and laparoscopic-assisted ERCP in patients with Roux-en-Y anatomy. Endoscopy 2019; 11: 1051-1058

[8] Law R, Leal C, Dayyeh BA et al. Role of immunosuppression in postendoscopic retrograde cholangiopancreatography pancreatitis after liver transplantation: A retrospective analysis. Liver Transpl 2013; 19 : 1354-1360

[9] Kedia P, Sharaiha RZ, Kumta NA et al. Internal EUS-directed transgastric ERCP (EDGE): game over. Gastroenterology 2014; 147: 566-568

[10] Wang BCM, Furnback W. Modelling the long-term outcomes of bariatric surgery: A review of cost-effectiveness studies. Best Pract Res Clin Gastroenterol 2013; 27: 987-995

[11] Martin A, Kistler CA, Wrobel P et al. Endoscopic ultrasound-guided pancreaticobiliary intervention in patients with surgically altered anatomy and inaccessible papillae: A review of current literature. Endosc Ultrasound 2016; 5: 149-156

[12] da Ponte-Neto AM, Bernardo WM, de A Coutinho LM et al. Comparison between enteroscopy-based and laparoscopy-assisted ERCP for accessing the biliary tree in patients with Roux-en-Y gastric bypass: systematic review and meta-analysis. Obes Surg 2018; 28: 40644076

[13] Dhindsa BS, Dhaliwal A, Mohan BP et al. EDGE in Roux-en-Y gastric bypass: How does it compare to laparoscopy-assisted and balloon enteroscopy ERCP: a systematic review and meta-analysis. Endosc Int Open 2020; 8: E163-E171

[14] Kedia P, Tarnasky PR, Nieto J et al. EUS-directed transgastric ERCP (EDGE) versus laparoscopy-assisted ERCP (LA-ERCP) for Roux-en-Y gastric bypass (RYGB) anatomy: a multicenter early comparative experience of clinical outcomes. J Clin Gastroenterol 2019; 53: 304-308

[15] Kedia P, Kumta NA, Widmer J et al. Endoscopic ultrasound-directed transgastric ERCP (EDGE) for Roux-en-Y anatomy: a novel technique. Endoscopy 2015; 47: 159-163

[16] James TW, Baron TH. Endoscopic ultrasound-directed transgastric ERCP (EDGE): a single-center US experience with follow-up data on fistula closure. Obes Surg 2019; 29: 451-456 